Tôhoku Math. Journ.

30 (1978), 1-12.

\title{
FAVARD'S SEPARATION THEOREM IN FUNCTIONAL DIFFERENTIAL EQUATIONS WITH INFINITE RETARDATIONS
}

\author{
YOSHIYUKI HINO
}

(Received July 19, 1976)

1. Introduction. Favard [1] has shown that if a linear almost periodic system

$$
\dot{x}(t)=A(t) x+f(t)
$$

has a bounded solution and if for every $B(t)$ in the hull $H(A)$, every nontrivial solution $x(t)$ of $\dot{x}(t)=B(t) x$ which is defined and bounded on $R$ (shortly, $R$-bounded) satisfies the condition

$$
\inf _{t \in R}\|x(t)\|>0,
$$

then system (1) has an almost periodic solution.

Recently, Kato [7] has pointed out that for functional differential equations the replacement of condition (2) in Favard's theorem by the condition

$$
\inf _{t \in R}\left(\sup _{\theta \in[-h, 0]}\|x(t+\theta)\|\right)>0
$$

is not obvious. However, Kato has shown that condition (2) can be replaced by condition (3) by considering a minimal solution with respect to a new norm $\|\cdot\|$ in $C\left([-h, 0], R^{n}\right)$ defined by

$$
\|\phi\|=\left(\int_{-h}^{0}\|\phi(s)\|^{2} d s\right)^{1 / 2}
$$

In this paper, more generally, we shall show that for functional differential equations with infinite retardations, we can replace condition (2) by the conditions

$$
\inf _{t \in R}\left(\sup _{\theta \in(-\infty, 0]}\|x(t+\theta)\| e^{\gamma \theta}\right)>0, \quad \gamma>0,
$$

and

$$
\inf _{t \in R}\left\{\sup _{\theta \in[-r, 0]}\|x(t+\theta)\|^{p}+\int_{-\infty}^{0}\|x(t+\theta)\|^{p} g(\theta) d \theta\right\}^{1 / p}>0
$$

by introducing semi-norm 


$$
\|\dot{\varphi}\|_{*}=\left\{\int_{-\infty}^{0}\|\dot{\phi}(\theta)\|^{2} e^{2 \gamma \theta} d \theta\right\}^{1 / 2}
$$

and

$$
\|\dot{\phi}\|_{*}= \begin{cases}\left\{\|\phi(0)\|^{2}+\int_{-\infty}^{0}\|\dot{\phi}(\theta)\|^{2} g(\theta) d \theta\right\}^{1 / 2}, & \text { if } \quad r=0, \\ \left\{\int_{-r}^{0}\|\dot{\phi}(\theta)\|^{2} d \theta+\int_{-\infty}^{0}\|\phi(\theta)\|^{2} g(\theta) d \theta\right\}^{1 / 2}, & \text { if } \quad r>0,\end{cases}
$$

for continuous and bounded functions $\phi$ mapping $(-\infty, 0]$ into $R^{n}$, respectively, where $g(\theta)$ is a nondecreasing positive function defined on $(-\infty, 0]$ such that $\int_{-\infty}^{0} g(\theta) d \theta<\infty$.

2. Hale's space and some lemmas. First we shall give a class of Banach spaces considered by Hale [2]. Let $x$ be any vector in $R^{n}$ and $\|x\|$ be the Euclidean norm of $x$. Let $B=B\left((-\infty, 0], R^{n}\right)$ be a space of functions mapping $(-\infty, 0]$ into $R^{n}$ with norm $\|\cdot\|_{B}$. For any $\phi$ in $B$ and any $\sigma$ in $[0, \infty)$, let $\phi^{\sigma}$ be the restriction of $\phi$ to the interval $(-\infty,-\sigma]$. This is a function mapping $(-\infty,-\sigma]$ into $R^{n}$. We shall denote by $B^{o}$ the space of such functions $\phi^{\sigma}$. For any $\eta \in B^{\sigma}$, we define the semi-norm $\|\eta !\|_{B^{\sigma}}$ of $\eta$ by

$$
\|\eta\|_{B^{\sigma}}=\inf \left\{\|\phi\|_{B}: \phi^{\sigma}=\eta\right\} .
$$

If $x$ is a function defined on $(-\infty, a), a>0$, then for each $t$ in $[0, a)$ we define the function $x_{t}$ by the relation $x_{t}(s)=x(t+s),-\infty<s \leqq 0$. For numbers $a$ and $\tau, a>\tau$, we denote by $A_{\tau}{ }^{a}$ the class of function $x$ mapping $(-\infty, a)$ into $R^{n}$ such that $x$ is a continuous function on $[\tau, a)$ and $x_{\tau} \in B$. The space $B$ is assumed to have the following properties:

( I ) $B$ is a Banach space.

(II) If $x$ is in $A_{\tau}{ }^{a}$, then $x_{t}$ is in $B$ for all $t$ in $[\tau, a)$ and $x_{t}$ is a continuous function of $t$, where $a$ and $\tau$ are constants such that $\tau<a \leqq \infty$.

(III) All bounded continuous functions mapping $(-\infty, 0]$ into $R^{n}$ are in $B$.

(IV) If a sequence $\left\{\phi_{k}\right\}, \phi_{k} \in B$, is uniformly bounded on $(-\infty, 0]$ with respect to the Euclidean norm $\|\cdot\|$ and converges to $\phi$ uniformly on any compact subset of $(-\infty, 0]$, then $\phi \in B$ and $\left\|\phi_{k}-\phi\right\|_{B} \rightarrow 0$ as $k \rightarrow \infty$.

REmark. Property (IV) is equivalent to the following property: For any $b>0$ and $\varepsilon>0$, there exist an $N>0$ and a $\delta>0$ such that

$$
\left\{\dot{\phi} \in B ;\|\phi\|_{B}<\varepsilon\right\} \supset\left\{\dot{\phi} \in B ; \sup _{\theta \in[-N, 0]}\|\phi(\theta)\|<\delta\right\} \cap\left\{\dot{\phi} \in B ; \sup _{\theta \in(-\infty, 0]}\|\phi(\theta)\|<b\right\} .
$$


(V) There are continuous, increasing and nonnegative functions $b(r)$, $c(r)$ defined on $[0, \infty), b(0)=c(0)=0$, such that

$$
\|\dot{\phi}\|_{B} \leqq b\left(\sup _{\theta \in[-\sigma, 0]}\|\dot{\phi}(\theta)\|\right)+c\left(\left\|\dot{\phi}^{\sigma}\right\|_{B^{\sigma}}\right)
$$

for any $\phi$ in $B$ and any $\sigma \geqq 0$.

(VI) If $\sigma$ is a nonnegative number and $\phi$ is an element in $B$, then $T_{\sigma} \dot{\phi}$ defined by $T_{\sigma} \phi(s)=\phi(s+\sigma), s \in(-\infty,-\sigma]$, is an element in $B^{\sigma}$ and $\left\|T_{\sigma} \dot{\phi}\right\|_{B^{\sigma}} \rightarrow 0$ as $\sigma \rightarrow \infty$.

In addition, we shall assume that the space $B$ has the following properties;

(VII) $B$ is separable.

(VIII) $\|\phi(0)\| \leqq M_{1}\|\phi\|_{B}$ for $M_{1}>0$.

In the following four lemmas, we assume that $f(t, \phi)$ is continuous in $(t, \phi) \in R \times B$ and almost periodic in $t$ uniformly for $\phi \in B$.

Lemma 1 (cf. Lemma 3 in [5]). Suppose that $f(t, \phi)$ satisfies the condition

$$
\sup \left\{\|f(t, \phi)\| ; t \in R,\|\phi\|_{B} \leqq \alpha\right\} \leqq F(\alpha)<\infty
$$

for every $\alpha>0$.

If the system

$$
\dot{x}(t)=f\left(t, x_{t}\right)
$$

has a solution $x(t)$ which is bounded on $[0, \infty)$, then for any $g(t, \phi)$ in $H(f)$ the system

$$
\dot{x}(t)=g\left(t, x_{t}\right)
$$

has an R-bounded solution. More exactly, if $\left\{x\left(t+t_{k}\right), f\left(t+t_{k}, \phi\right)\right\}$ converges to $(y(t), g(t, \dot{\rho}))$, then $y(t)$ is a bounded solution of $(6)$ on $\left(-\varlimsup_{k \rightarrow \infty} t_{k}, \infty\right)$.

The following lemma can be proved by slightly modifying the proof of Lemma 1 in [6].

LEMMA 2. If $f(t, \phi)$ is linear in $\phi$, then it satisfies condition (4) with $F(\alpha)=L \alpha$ for $a$ constant $L>0$.

For continuous and bounded function $\phi$ mapping $(-\infty, 0]$ into $R^{n}$, let $\|\dot{\phi}\|_{*}$ be a semi-norm which has the following properties:

(a) For any $d>0$, there exists an $M(d)>0$ such that if $\|\phi(t)\| \leqq d$ for all $t \in(-\infty, 0]$, then $\|\phi\|_{*} \leqq M(d)$.

(b) If a sequence $\left\{\phi_{k}\right\}$ is continuous and uniformly bounded on $(-\infty, 0]$ with respect to the Euclidean norm $\|\cdot\|$ and converges to $\dot{\rho}$ uni- 
formly on any compact subset of $(-\infty, 0]$, then $\left\|\dot{\phi}_{k}-\phi\right\|_{*} \rightarrow 0$ as $k \rightarrow \infty$.

(c) There exists a $\beta(\alpha)$ such that if $x(t)$ is an $R$-bounded solution of (5) and satisfies $\left\|x_{t}\right\|_{*} \leqq \alpha, \alpha>0$, where $f(t, \phi)$ satisfies condition (4) with $F(\alpha)=o\left(\alpha^{3}\right)$ as $\alpha \rightarrow \infty$, then $\|x(t)\| \leqq \beta(\alpha)$. and 4 .

Existence of such a semi-norm $\|\cdot\|_{*}$ will be discussed in Sections 3

For an $R$-bounded and continuous function $x(t)$, put

$$
\lambda(x)=\sup \left\{\left\|x_{t}\right\|_{*} ; t \in R\right\} .
$$

Lemma 3. Suppose that $f(t, \phi)$ satisfies condition (4) and that system (5) has an R-bounded solution. Let $\Lambda(f)$ be defined by

$$
\Lambda(f)=\inf \{\lambda(x) ; x(t) \text { is an R-bounded solution of }(5)\} .
$$

Then for every $g(t, \phi) \in H(f)$, we have $\Lambda(g)=\Lambda(f)$.

Proof. First of all, we note that $\lambda(x)<\infty$ if $x(t)$ is $R$-bounded by property (a). For every $\varepsilon>0$, there exists an $R$-bounded solution of (5) such that $\lambda(x) \leqq A(f)+\varepsilon$. Since $x(t)$ is an $R$-bounded solution of (5), for every $g(t, \phi) \in H(f)$, system (6) has a solution $y(t)$ to which $\left\{x\left(t+t_{k}\right)\right\}$ converges uniformly on any compact interval in $R$ for some sequence $\left\{t_{k}\right\}$ by Lemma 1 . Then

$$
\left\|y_{t}\right\|_{*}-\left\|x_{t+t_{k}}\right\|_{*} \leqq\left\|x_{t+t_{k}}-y_{t}\right\|_{*} \rightarrow 0 \quad \text { as } \quad k \rightarrow \infty
$$

by property (b). This implies

$$
\Lambda(g) \leqq \lambda(y) \leqq \lambda(x) \leqq \Lambda(f)+\varepsilon,
$$

and hence $\Lambda(g) \leqq \Lambda(f)$. On the other hand, $g(t, \phi) \in H(f)$ is almost periodic uniformly for $\phi \in B$ and $f(t, \phi) \in H(g)$, and hence $\Lambda(f) \leqq \Lambda(g)$. Thus we have $\Lambda(g)=\Lambda(f)$ for every $g(t, \phi) \in H(f)$.

LEMMA 4. Suppose that $f(t, \phi)$ satisfies condition (4) with $F(\alpha)=o\left(\alpha^{3}\right)$ as $\alpha \rightarrow \infty$ and that system (5) has an $R$-bounded solution. Then there exists an $R$-bounded solution $x(t)$ of (5) with the property $\lambda(x)=\Lambda(f)$.

Proof. By the definition of $A(f)$, there exists a sequence $\left\{x^{k}(t)\right\}$ of $R$-bounded solution of (5) such that $\lambda\left(x^{k}\right) \leqq \Lambda(f)+1 / k \leqq \Lambda(f)+1$. Since $\|\left. x_{t}^{k}\right|_{*} \leqq \Lambda(f)+1$, there exists a $\beta>0$ such that $\left\|x^{k}(t)\right\|<\beta$ for all $k$ and all $t \in R$ by property (c). Let $K$ be such that

$$
\begin{aligned}
K= & \left\{\phi \in B ;\|\phi(\theta)\| \leqq \beta \text { on } \theta \in(-\infty, 0],\left\|\phi\left(\theta_{1}\right)-\dot{\phi}\left(\theta_{2}\right)\right\| \leqq F(b(\beta))\left|\theta_{1}-\theta_{2}\right|,\right. \\
& \left.\theta_{1}, \theta_{2} \in(-\infty, 0]\right\},
\end{aligned}
$$

where $b(\cdot)$ is the one given in property $(\mathrm{V})$ of the space $B$. Clearly, $K$ is a compact subset of $B$. Since $\left\|\dot{x}^{k}(t)\right\| \leqq F(b(\beta))$ for all $k$ and all 
$t, x^{k}{ }_{t} \in K$ for all $k$ and all $t \in R$. Thus $\left\{x^{k}(t)\right\}$ has a subsequence $\left\{x^{k j}(t)\right\}$ which converges to an $R$-bounded solution $x(t)$ of (5). On the other hand, by using the same arguments as in the proof of Lemma 3, we have $\lambda(x) \leqq \Lambda(f)$. That is, $\lambda(x)=\Lambda(f)$.

3. The space $\mathscr{C}$ with norm $\sup _{\theta \in(-\infty, 0]}\|\phi(\theta)\| e^{r \theta}$. The following class of Banach spaces has been discussed by Hino in [4] as one of Hale's spaces.

DEFINITION 1. The space $\mathscr{C}$ consists of all continuous functions mapping $(-\infty, 0]$ into $R^{n}$ such that $\phi(\theta) e^{r \theta} \rightarrow 0$ as $\theta \rightarrow-\infty$ with norm $\|\dot{ }\|_{\mathscr{\varepsilon}}=\sup _{\theta \in\{-\infty, 0]}\|\phi(\theta)\| e^{\gamma \theta}, \gamma>0$.

It is easily seen that the space $\mathscr{C}$ has properties $(\mathrm{I}) \sim(\mathrm{VIII})$.

For bounded functions $\phi$ in $\mathscr{C}$, if we define $\|\phi\|_{*}$ by

$$
\|\boldsymbol{\phi}\|_{*}=\left\{\int_{-\infty}^{0}\|\phi(\theta)\|^{2} e^{2 \gamma \theta} d \theta\right\}^{1 / 2}
$$

then it has properties (a), (b), and (c). It is clear that it has properties (a) and (b). We shall show that it has property (c). Assume that $x(t)$ is an $R$-bounded solution of (5) and $\left\|x_{t}\right\|_{*} \leqq \alpha, \alpha>0$. Clearly, for any $T>0$

$$
e^{-r T}\left(\int_{-T}^{0}\|x(t+\theta)\|^{2} d \theta\right)^{1 / 2} \leqq\left(\int_{-T}^{0}\|x(t+\theta)\|^{2} e^{2 \gamma \theta} d \theta\right)^{1 / 2} \leqq\left\|x_{t}\right\|_{*} \leqq \alpha,
$$

and hence property (c) follows from Lemma 2 and the following lemma.

LEMma 5 (cf. Lemma 4 in [7]). Suppose that $f(t, \phi)$ satisfies condition (4) with $F(\alpha)=o\left(\alpha^{3}\right)$ as $\alpha \rightarrow \infty$. Then for any $\alpha>0$, there exists $\alpha$ constant $\beta>0$ such that if $x(t)$ is an R-bounded solution of system (5) and satisfies $\sup _{t \in R}\left(\int_{-T}^{0}\|x(t+\theta)\|^{2} d \theta\right)^{1 / 2} \leqq \alpha$ for some $T>0$, we have $\|x(t)\| \leqq \beta$ for all $t \in R$.

Here we should note that this $\|\cdot\|_{*}$ has the following property;

(d) If $x^{1}(t)$ and $x^{2}(t)$ are $R$-bounded continuous functions, then

$$
\left\{\left\|x_{t}^{1}\right\|_{*}^{2}+\left\|x_{t}^{2}\right\|_{*}^{2}\right\} / 2=\left\|y_{t}\right\|_{*}^{2}+\left\|z_{t}\right\|_{*}^{2},
$$

where $y(t)=\left\{x^{1}(t)+x^{2}(t)\right\} / 2$ and $z(t)=\left\{x^{1}(t)-x^{2}(t)\right\} / 2$.

4. The space $\mathscr{S}$ with norm $\left\{\left(\sup _{\theta \in[-r, 0]}\|\phi(\theta)\|\right)^{p}+\int_{-\infty}^{0}\|\phi(\theta)\|^{p} g(\theta) d \theta\right\}^{1 / p}$.

We shall discuss a class of Banach spaces considered by Naito in [8] as one of Hale's spaces.

Definition 2. Let $r \geqq 0, p \geqq 1$, and let $g(\theta)$ be a nondecreasing 
positive function defincd on $(-\infty, 0]$ such that $\int_{-\infty}^{0} g(\theta) d \theta<\infty$. The space $\mathscr{B}$ consists of all functions $\phi$ mapping $(-\infty, 0]$ into $R^{n}$, which are Lebesgue measurable on $(-\infty, 0]$ and are continuous on $[-r, 0]$ with norm $\|\dot{\phi}\|_{\circledast}=\left\{\left(\sup _{\theta \in i-r, 0]}\|\dot{\phi}(\theta)\|\right)^{p}+\int_{-\infty}^{0}\|\dot{\phi}(\theta)\|^{p} g(\theta) d \theta\right\}^{1 / p} \cdot$ When $r=0$, we do not assume the continuity of $\phi$ at $\theta=0$.

It is easily shown that the space $\mathscr{B}$ also has properties $(\mathrm{I}) \sim(\mathrm{VIII})$.

For continuous and bounded function $\phi$ mapping $(-\infty, 0]$ into $R^{n}$, we can consider

$$
\|\phi\|_{*}= \begin{cases}\left\{\|\phi(0)\|^{2}+\int_{-\infty}^{0}\|\phi(\theta)\|^{2} g(\theta) d \theta\right\}^{1 / 2} & \text { if } \quad r=0, \\ \left\{\int_{-r}^{0}\|\phi(\theta)\|^{2} d \theta+\int_{-\infty}^{0}\|\phi(\theta)\|^{2} g(\theta) d \theta\right\}^{1 / 2} & \text { if } \quad r>0\end{cases}
$$

which has properties (a), (b), (c), and (d). It is clear that it has properties (a), (b), and (d). Assume that $x(t)$ is an $R$-bounded solution of (5) and $\left\|x_{t}\right\|_{*} \leqq \alpha, \alpha>0$. If $r=0$, then it satisfies

$$
\|x(t)\| \leqq\left\|x_{t}\right\|_{*} \leqq \alpha
$$

Since

$$
\left(\int_{-r}^{0}\|x(t+\theta)\|^{2} d \theta\right)^{1 / 2} \leqq\left\|x_{t}\right\|_{*} \leqq \alpha, \quad \text { if } \quad r>0,
$$

property (c) follows from Lemma 5.

5. Existence theorem for almost periodic solutions of linear systems.

LEMмA 6. Let $r>0$ and $\phi(\theta)$ be defined on $[-r, 0]$. If $\phi(\theta)$ satisfies a Lipschitz condition

$$
|| \phi\left(\theta_{1}\right)-\phi\left(\theta_{2}\right) \| \leqq L\left|\theta_{1}-\theta_{2}\right|, \quad \theta_{1}, \theta_{2} \in[-r, 0],
$$

then

$$
\left(\int_{-r}^{0}\|\phi(\theta)\|^{2} d \theta\right)^{1 / 2} \geqq\left\{\min \left(r / 3,\left(\sup _{\theta \in[-r, 0]}\|\phi(\theta)\|\right) / 3 L\right)\right\}^{1 / 2} \times\left(\sup _{\theta \in[-r, 0]}\|\phi(\theta)\|\right) .
$$

For the proof, see ([7], pp 87-88).

Theorem. Suppose that $A(t, \phi)$ is continuous in $(t, \phi) \in R \times \mathscr{C}$ $(R \times \mathscr{B})$, linear in $\phi$ and almost periodic in $t$ uniformly for $\phi \in \mathscr{C}(\mathscr{B})$, and that

(*) for every $B(t, \phi) \in H(A)$, every nontrivial $R$-bounded solution of the system 


$$
\dot{x}(t)=B\left(t, x_{t}\right)
$$

satisfies the condition

$$
\inf _{t \in R}\left\|x_{t}\right\|_{\mathscr{C}}>0 \quad\left(\inf _{t \in R}\left\|x_{t}\right\|_{\mathscr{B}}>0\right) .
$$

Then for any almost periodic function $f(t)$, the system

$$
\dot{x}(t)=A\left(t, x_{t}\right)+f(t)
$$

has an almost periodic solution, whenever it has a bounded solution on $[0, \infty)$.

Proof. There exists an $R$-bounded solution $x(t)$ of (9) with the minimal semi-norm $\lambda(x)$ by Lemmas 2 and 4 .

Now we shall show that for each $B(t, \phi)+g(t) \in H(A+f)$, the system

$$
\dot{x}(t)=B\left(t, x_{t}\right)+g(t)
$$

has a unique $R$-bounded solution with the minimal semi-norm.

Let $x^{1}(t)$ and $x^{2}(t)$ be $R$-bounded solutions of (10) with the minimal semi-norm. Clearly, $z(t)=\left\{x^{1}(t)-x^{2}(t)\right\} / 2$ is a solution of the homogeneous system (7) and $y(t)=\left\{x^{1}(t)+x^{2}(t)\right\} / 2$ is a solution of system (10). By property $(d)$, we have

$$
\left\{|| x_{t}^{1}\left\|_{*}^{2}+\right\| x_{t}^{2} \|_{*}^{2}\right\} / 2=\left\|y_{t}\right\|_{*}^{2}+\left\|z_{t}\right\|_{*}^{2},
$$

which implies

$$
\inf _{t \in R}\left\|z_{t}\right\|_{*}=0 \text {. }
$$

Assume that $\sup _{t \in R}\|z(t)\|=\delta>0$. Clearly, $\delta<\infty$. Then there exists an $L_{1}>0$ such that $\sup _{t \in R}\left\|B\left(t, z_{t}\right)\right\| \leqq L_{\perp}$ by property (V) and Lemma 2.

(i) The case where the space is $\mathscr{C}$. The relation (11) implies that for any $\varepsilon>0$, there exists a $t_{0} \in R$ such that

$$
\left\|z_{t_{0}}\right\|_{*}=\left\{\int_{-\infty}^{0}\left\|z\left(t_{0}+\theta\right)\right\|^{2} e^{2 \gamma \theta} d \theta\right\}^{1 / 2}<\varepsilon .
$$

There exists a $T>1$ such that

$$
\sup _{\theta \in(-\infty,-T]}\left\|z\left(t_{0}+\theta\right)\right\| e^{\gamma \theta} \leqq \delta e^{-\gamma T}<\varepsilon .
$$

Since

$$
\left\|z\left(t+\theta_{1}\right) e^{r \theta_{1}}-z\left(t+\theta_{2}\right) e^{r \theta_{2}}\right\| \leqq L_{2}\left|\theta_{1}-\theta_{2}\right| \quad \text { for } \quad \theta_{1}, \theta_{2} \in[-T, 0],
$$

where $L_{2}=L_{1}+\gamma \delta$, it follows from (12) and Lemma 6 that 


$$
\begin{aligned}
\varepsilon^{2} & >\int_{-T}^{0}\left\|z\left(t_{0}+\theta\right) e^{\gamma \theta}\right\|^{2} d \theta \\
& \geqq \min \left\{T / 3,\left(\sup _{\theta \in[-T, 0]}\left\|z\left(t_{0}+\theta\right) e^{\gamma \theta}\right\|\right) / 3 L_{2}\right\} \times\left(\sup _{\theta \in[-T, 0]}\left\|z\left(t_{0}+\theta\right) e^{\gamma \theta}\right\|\right)^{2} .
\end{aligned}
$$

Hence we have

$$
\sup _{\theta \in[-T, 0]}\left\|z\left(t_{0}+\theta\right) e^{\gamma \theta}\right\| \leqq \max \left\{\sqrt{3 \varepsilon}, \sqrt[3]{3 L_{2} \varepsilon^{2}}\right\},
$$

because $T>1$. By (13) and (14), we have $\inf _{t \in R}\left\|z_{t}\right\|_{c}=0$, which contradicts to condition (8). Thus $z(t)=0$ on $R$.

(ii) The case where the space is $\mathscr{B}$. Define $\left\|z_{t}\right\|_{* *}$ by

$$
\left\|z_{t}\right\|_{* *}=\sup _{\theta \in[-r, 0]}\|z(t+\theta)\|+\left(\int_{-\infty}^{0}\|z(t+\theta)\|^{p} g(\theta) d \theta\right)^{1 / p} .
$$

Then, we have

$$
\left\|z_{t}\right\|_{s s} \leqq\left\|z_{t}\right\|_{* *} \cdot
$$

It follows from (11) that for any $\varepsilon>0$, there exists a $t_{0} \in R$ such that $\left\|z_{t_{0}}\right\|_{*}<\varepsilon$, that is,

$$
\begin{cases}\left\|z\left(t_{0}\right)\right\|<\varepsilon & \text { if } r=0 \\ \left(\int_{-r}^{0}\left\|z\left(t_{0}+\theta\right)\right\|^{2} d \theta\right)^{1 / 2}<\varepsilon & \text { if } \quad r>0\end{cases}
$$

and

$$
\left(\int_{-\infty}^{0}\left\|z\left(t_{0}+\theta\right)\right\|^{2} g(\theta) d \theta\right)^{1 / 2}<\varepsilon .
$$

(ii.1) The case where $1 \leqq p<2$. By Hölder's inequality, we have

$$
\begin{aligned}
& \left(\int_{-\infty}^{0}\|z(t+\theta)\|^{p} g(\theta) d \theta\right)^{1 / p}=\left(\int_{-\infty}^{0}\|z(t+\theta)\|^{p} g(\theta)^{p / 2} g(\theta)^{1-p / 2} d \theta\right)^{1 / p} \\
& \quad \leqq\left\{\left(\int_{-\infty}^{0}\|z(t+\theta)\|^{2} g(\theta) d \theta\right)^{p / 2} \times\left(\int_{-\infty}^{0}\left(g(\theta)^{1-p / 2}\right)^{2 /(2-p)} d \theta\right)^{(2-p) / 2}\right\}^{1 / p} \\
& \quad \leqq\left(\int_{-\infty}^{0}\|z(t+\theta)\|^{2} g(\theta) d \theta\right)^{1 / 2} \times\left(\int_{-\infty}^{0} g(\theta) d \theta\right)^{(2-p) / p},
\end{aligned}
$$

because $1<2 / p$. By Lemma 6 , it holds that

$$
\begin{aligned}
\left(\int_{-r}^{0}\|z(t+\theta)\|^{2} d \theta\right)^{1 / 2} \geqq & \left\{\min \left(r / 3,\left(\sup _{\theta \in[-r, 0]}\|z(t+\theta)\|\right) / 3 L_{1}\right)\right\}^{1 / 2} \\
& \times\left(\sup _{\theta \in[-r, 0]}\|z(t+\theta)\|\right),
\end{aligned}
$$

if $r>0$. By (16), (17), (18), and (19), we have 


$$
\left\|z_{t_{0}}\right\|_{* *} \leqq \begin{cases}\varepsilon+\left(\int_{-\infty}^{0} g(\theta) d \theta\right)^{(2-p) / p} \times \varepsilon & \text { if } \quad r=0, \\ \max \left\{\sqrt{3 \varepsilon / r}, \sqrt[3]{3 L_{1} \varepsilon^{2}}\right\}+\left(\int_{-\infty}^{0} g(\theta) d \theta\right)^{(2-p) / p} \times \varepsilon & \text { if } \quad r>0,\end{cases}
$$

which implies that $\inf _{t \in R}\left\|z_{t}\right\|_{* *}=0$. Therefore $\inf _{t \in R}\left\|z_{t}\right\|_{\infty}=0$ by (15), which contradicts to condition (8). Thus $z(t)=0$ on $R$.

(ii.2) The case which $p \geqq 2$. It is easily seen that

$$
\left(\int_{-\infty}^{0}\|z(t+\theta)\|^{2} g(\theta) d \theta\right)^{1 / p} \geqq\left\{\left(\int_{-\infty}^{0}\|z(t+\theta)\|^{p} g(\theta) d \theta\right) / \delta^{p-2}\right\}^{1 / p},
$$

because $p \geqq 2$. By (16), (17), (19), and (20), we have

$$
\left\|z_{t_{0}}\right\|_{* *} \leqq \begin{cases}\varepsilon+\delta^{(p-2) / p} \times \varepsilon^{2 / p} & \text { if } r=0, \\ \max \left\{\sqrt{3 \varepsilon / r}, \sqrt[3]{3 L_{1} \varepsilon^{2}}\right\}+\delta^{(p-2) / p} \times \varepsilon^{2 / p} & \text { if } r>0,\end{cases}
$$

which implies that $\inf _{t \in R}\left\|z_{t}\right\|_{* *}=0$. Therefore $\inf _{t \in R}\left\|z_{t}\right\|_{s}=0$ by (15), which contradicts to condition (8). Thus $z(t)=0$ on $R$. Thus system (10) has a unique $R$-bounded solution with the minimal semi-norm.

Let $p(t)$ be the solution of (9) with the minimal semi-norm. It is easy to see that if $(y, C(t, \phi)+h) \in H(p, A+f), y(t)$ is the solution of the system

$$
\dot{x}(t)=C\left(t, x_{t}\right)+h(t)
$$

with the minimal semi-norm by Lemma 3 . Let $\left\{\tau_{k}\right\}$ be a sequence such that $A\left(t+\tau_{k}, \phi\right)+f\left(t+\tau_{k}\right) \rightarrow B(t, \phi)+g(t)$ uniformly on $R \times S$ as $k \rightarrow \infty$, where $S$ is any compact subset of $\mathscr{C}(\mathscr{B})$. Suppose that $p\left(t+\tau_{k}\right)$ is not uniformly convergent on $R$. Then, by the same idea as in the proof of Theorem 5 in [5], we can find two solutions $\eta(t) \in H(p)$ and $\zeta(t) \in H(p)$ of some system in the hull $H(A+f)$ which satisfies

$$
\left\|\eta_{0}-\zeta_{0}\right\|_{*}>\varepsilon
$$

for some $\varepsilon>0$. Thus we can find two minimal solutions of some system in the hull. This contradicts the uniqueness of the minimal solution. Thus we see that $p(t)$ is an almost periodic solution of (9). This completes the proof.

REMARK. If we define a number $\beta$ by

$$
\beta=\inf \left\{\operatorname{Re} \lambda: \int_{-\infty}^{0}\left|e^{\lambda \theta}\right|^{p} g(\theta) d \theta<\infty\right\},
$$

where $g(\theta)$ is the one given in Definition 2, then $\beta$ is clearly nonpositive. If $\beta \neq 0$, we can regard our theorem with the space $\mathscr{B}$ as a corollary of our theorem with the space $\mathscr{C}$. Furthermore, we can 
replace the assumption $(*)$ in our theorem with $\mathscr{B}$ by the following assumption:

(**) there exists a $\gamma, \beta<-\gamma<0$, such that for every $B(t, \dot{\phi}) \in H(A)$, every nontrivial $R$-bounded solution of system (7) satisfies the condition

$$
\inf _{t \in R}\left\|x_{t}\right\|_{\mathscr{\sigma}}>0 \text {, }
$$

where $\left\|x_{t}\right\|_{\mathscr{C}}=\sup _{\theta \in\{-\infty, 0]}\|x(t+\theta)\| e^{\gamma \theta}$.

In fact, for the number $\gamma$ in $(* *)$, the space $\mathscr{C}$ is naturally and continuously imbeded into $\mathscr{B}$, that is, there exists a constant $d(\gamma)$ such that

$$
\|\phi\|_{\infty \phi} \leqq d(\gamma)\|\phi\|_{\mathscr{C}} \quad \text { for } \quad \phi \in \mathscr{C}
$$

(cf. Lemma 3.3 in [9]). Let $A(t, \phi)$ be a function defined on $R \times \mathscr{B}$ satisfying the assumptions in our theorem with $\mathscr{B}$. Conditions (22) and (23) imply that the restriction $\widetilde{A}$ of $A$ on $R \times \mathscr{C}$ satisfies the assumptions in our theorem with $\mathscr{C}$. Suppose that $f(t)$ is an almost periodic function for which system (9) has a bounded solution on $[0, \infty)$. By Lemma 1 , system (9) has an $R$-bounded solution, which is obviously an $R$-bounded solution of the system

$$
\dot{x}(t)=\widetilde{A}\left(t, x_{t}\right)+f(t) .
$$

Then, Theorem with $\mathscr{C}$ says that system (24) has an almost periodic solution $p(t)$. Since $\widetilde{A}\left(t, p_{t}\right)=A\left(t, p_{t}\right)$ for $t \in R, p(t)$ is a solution of system (9).

In the same ways as above, we can replace the condition (3) in [7] by the condition $(* *)$, where $\beta$ is assumed to be $-\infty$.

6. Autonomous linear system. Consider an autonomous linear system

$$
\dot{x}(t)=A\left(x_{t}\right),
$$

where $A(\phi)$ is a bounded linear operator on $\mathscr{B}$ into $R^{n}$. We assume that the function $g(\theta)$ in Definition 2 satisfies the condition

$$
g\left(\theta_{1}+\theta_{2}\right) \leqq g\left(\theta_{1}\right) g\left(\theta_{2}\right) \quad \text { for } \quad \theta_{1}, \theta_{2} \in(-\infty, 0] .
$$

Then it has been proved by Naito (Theorem 4.4 in [8]) that there exist two positively invariant spaces $S$ and $U$ such that

$$
\mathscr{B}\left((-\infty, 0], R^{n}\right)=S \oplus U
$$

with the properties that

(i) every solution of (25) starting from $S$ tends to zero as $t \rightarrow \infty$, 
(ii) $\operatorname{dim} U<\infty$, and the solutions of (25) starting from $U$ are governed by an autonomous linear system of ordinary differential equations all of whose eigenvalues have nonnegative real part.

Hence, by the same arguments as in ([7], p. 91), we can show that if $x(t)$ is a nontrivial $R$-bounded solution of (25), then it satisfies condition (8).

REMARK. In order to show that the above decomposition of the space $\mathscr{B}$ according to Theorem 4.4 in [8], we must see that the condition

$$
\beta<0
$$

holds, where $\beta$ is the one defined by (21). However, Professor Naito informed me that condition (27) follows from condition (26). I represent here a method due to Professor Naito. If condition (26) holds, then there exists a number $\alpha$ such that

$$
\alpha=\sup _{\theta<0}(\log g(\theta)) / \theta=\lim _{\theta \rightarrow-\infty}(\log g(\theta)) / \theta,
$$

(cf. Theorem 7.6.1 in [3]). It is clear that $g(\theta) \geqq e^{\alpha \theta}$ for $\theta \in(-\infty, 0]$ and that for any $\gamma<\alpha$, there exists a constant $N(\gamma)$ such that $g(\theta) \leqq N(\gamma) e^{\gamma \theta}$ for $\theta \in(-\infty, 0]$. Since $g(\theta)$ is nondecreasing and integrable, it holds that $0<\alpha \leqq \infty$. Hence we have the relation

$$
\beta=-\alpha / p
$$

where $p$ is the one given in Definition 2, which implies condition (27).

Finally the author thanks Professors T. Naito and T. Furumochi for their invaluable conversations and comments.

\section{REFERENCES}

[1] J. Favard, Leçons sur les fonctions presque-périodiques, Gauthier-Villars, Paris, 1933.

[2] J. K. Hale, Dynamical systems and stability, J. Math. Anal. Appl., 26 (1969), 39-59.

[3] E. Hill ANd R. S. PhIllips, Functional analysis and semi-groups, Colloq. Publ. Amer. Math. Soc., 1957.

[4] Y. Hino, Asymptotic behavior of solutions of some functional differential equations, Tôhoku Math. J., 22 (1970), 98-108.

[5] Y. Hino, Stability and existence of almost periodic solutions of some functional differential equations, Tôhoku Math. J., 28 (1976), 389-409.

[6] J. КАто, Remarks on linear functional differential equations, Funkcial. Ekvac., 12 (1969), 89-98.

[7] J. KAто, Favard's separation theorem in functional differential equations, Funkcial. Ekvac., 18 (1975), 85-98.

[8] T. NAITo, On autonoumous linear functional differential equations with infinite retardations, J. Differential Equs., 21 (1976), 297-315. 
[9] T. NAITo, Adjoint equations of autonomous linear functional differential equations with infinite retardations, Tôhoku Math. J., 28 (1976), 135-143.

Department of Mathematics

IWATE UNIVERSITY

MORIOKA, JAPAN 